\title{
Understanding the spectra of isolated Herbig stars in the frame of a passive disk model
}

\author{
C. Dominik ${ }^{1}$, C. P. Dullemond ${ }^{2}$, L. B. F. M. Waters ${ }^{1,3}$, and S. Walch ${ }^{2}$ \\ 1 Sterrenkundig Instituut "Anton Pannekoek", Kruislaan 403, 1098 SJ Amsterdam, The Netherlands \\ e-mail: dominik@science.uva.nl \\ 2 Max Planck Institut für Astrophysik, Karl Schwarzschild Strasse 1, 85748 Garching, Germany \\ e-mail: dullemon@mpa-garching .mpg. de \\ 3 Instituut voor Sterrenkunde, Katholieke Universiteit Leuven, Celestijnenlaan 200 B, 3001 Heverlee, Belgium
}

Received 26 July 2002 / Accepted 7 November 2002

\begin{abstract}
We discuss spectral energy distributions of a sample of Herbig Ae/Be stars in the context of a passive irradiated disk model. The data have been presented earlier by Meeus et al. (2001), and preliminary interpretations of these data were given in that paper. While the spectra of Herbig Ae stars all show similarities, there is significant variation between the spectra, in particular in the shape of the mid-IR rise and in the presence or absence of a silicate feature. We explore the hypothesis that all these different spectra can be interpreted as pure disk spectra without additional components. Using the model of Dullemond et al. (2001) we deduce the disk parameters of a number of the sources, and find that for a large fraction of investigated sources, satisfactory fits can be obtained. The derived model parameters show that some group Ia sources can only be fit with radially increasing surface densities, indicating the presence of depleted inner disk regions. The steep-sloped SEDs of group IIa sources can be fit with very compact disks, probably representing disks with collapsed outer regions. The largest difficulties arise from sources that do not show significant silicate emission features. Our attempts to explain these objects with a pure geometric effect are only partially successful. It seems that these stars indeed require a strong depletion of small silicate grains.
\end{abstract}

Key words. stars: circumstellar matter - infrared: stars

\section{Introduction}

Herbig $\mathrm{Ae} / \mathrm{Be}$ stars (herafter referred to as HAEBE stars) are thought to be young stellar objects of intermediate mass (Herbig 1960; Strom et al. 1972; Finkenzeller \& Mundt 1984; The et al. 1994; Malfait et al. 1998). They are associated with large amounts of circumstellar matter, both gas and dust, and are found in or near star forming regions. In the seminal paper of Herbig (1960), HAEBEs were defined to be near a reflection nebulosity, because this ensured they are young and close to a star forming region. The close proximity to molecular cloud material in combination with limited angular resolution observations makes it hard to distinguish dust and gas emission from the loose surroundings from that of the circumstellar disk. However, objects have been found that are very similar to other HAEBEs but lack a reflection nebulosity (see e.g. Waters \& Waelkens 1998). These stars are called isolated Herbig stars. Hipparcos observations of the nearest HAEBE stars have confirmed their pre-main-sequence nature (van den Ancker et al. 1997).

Send offprint requests to: C. Dominik, e-mail: dominik@science.uva.nl
HAEBE stars have a strong infrared (IR) excess due to circumstellar dust, often carrying a considerable fraction of the total luminosity of the system. Mid-IR imaging has shown that some HAEBE stars have spatially extended emission at these wavelengths (Prusti et al. 1994; di Francesco et al. 1994; di Francesco et al. 1998); these stars often (but not always) are of (early) $B$ spectral type, and their emission can be understood in terms of heating of the surrounding molecular cloud by the strong UV radiation field of the young star, possibly in combination with a disk (Miroshnichenko et al. 1999; Henning et al. 1998). However, the less luminous late B, and A type stars in the HAEBE class often show unresolved or compact emission in the mid-IR when observed with 4 m class telescopes on a scale of 1-2 arcsec, corresponding to 100-200 AU at typical distances of $100 \mathrm{pc}$. Van den Ancker et al. (private communication) have unsuccessfully tried to resolve several of the sources in the sample (HD 100546, HD 104237, HD 144432, HD 163296) with the ESO 3.6 m telescope, finding only upper limit of typically $0.7^{\prime \prime}$. Other observations have succeeded resolving the emission of some stars (e.g. AB Aurigae (Marsh et al. 1995), HD 100546 (Grady et al. 2001a), HD 97048 (van Boeckel et al., in preparation)) and found compact sizes. The isolated Herbig stars usually show little or no reddening 
at optical wavelengths, which is remarkable given their large $L_{\mathrm{IR}} / L_{*}$ ratio. An obvious way to explain this observation is to assume a flattened, disk-like distribution of the gas and dust viewed at some intermediate inclination angle. This disk can be understood as the passively heated remnant of the accretion disk which was present during the initial phase of star formation, and is similar to disks seen around the lower mass T Tauri stars. Since such passive disks are believed to be the site of planet formation, HAEBE stars have gained considerable attention in recent years.

Obviously, the best way to determine the geometry of the circumstellar material around isolated HAEBE stars is by direct imaging. Mannings \& Sargent (1997) spatially resolved the millimeter continuum and line emission from the Herbig Ae star HD 163296 and found an elongated structure. Submillimeter aperture synthesis images in CO lines show rotation profiles in a number of Herbig Ae stars, with rotational velocities consistent with a Keplerian disk (Dutrey et al. 1998; Qi et al. 1999; Mannings \& Sargent 1997; Mannings \& Sargent 2000). Based on this evidence one may conclude with some confidence that at least the outer parts of the circumstellar matter distribution around some (isolated) Herbig Ae stars are, just as in the case of $\mathrm{T}$ Tauri stars, in fact rotating circumstellar disks.

Observations of the spatial distribution of the circumstellar matter closer to the star (inwards of about $100 \mathrm{AU}$ ) have not yet resulted in a clear picture. There seems to be conflicting evidence suggesting both a flattened and more spherical distribution of gas and dust. Near-IR and optical imaging using ground-based adaptive optics and HST in several cases shows the emission to be flattened (Grady et al. 2000; Grady et al. 2001b; Grady et al. 2001a; Pantin et al. 2000). However, interferometric observations at $2 \mu \mathrm{m}$, probing the inner regions on scales below $10 \mathrm{AU}$, have shown little or no evidence for a disk-like geometry (Millan-Gabet et al. 2001).

In view of the scarcity of resolved observations at these spatial scales, it seems that the procedure of fitting a model to the observed SED is still a valuable tool to study the properties of circumstellar matter around young stars. Under the assumption that the circumstellar dust is in radiative equilibrium, the temperature of the dust can be calculated by solving the radiative transfer equation. By varying the density distribution and the dust composition, one can eventually find a good "fit" to the data. One has to keep in mind however that this procedure can not uniquely determine the geometry and density distribution of the circumstellar matter. Often, a multitude of different parameter sets lead to equally good fits (Thamm et al. 1994). This clearly means that the SED alone does not contain enough information about the geometry and dust distribution in these objects.

Nevertheless, model fitting can retrieve interesting information from the SED if one uses additional constraints to the density distribution. These constraints can be obtained from e.g. spectroscopic quantities (lines, features), or any kind of imaging data when available. In addition to this, one can add physics to the model. For instance, one could require the density distribution to be in hydrostatic equilibrium: a self-consistent disk model. A simple, but powerful model of this kind is the semi-analytic passive flaring disk model of Chiang \& Goldreich (1997). This model naturally reproduces the shape of the spectrum of many Herbig Ae stars longwards of 6 micron. Moreover, as was first noted by Natta et al. (2001), if one correctly accounts for emission from the disk's inner rim, also the SED shortwards of 6 microns (the conspicuous 3 micron bump) can be explained. In a recent paper, Dullemond et al. (2001, henceforth DDN), presented such a self-consistent disk model including both the emission from the flaring part of the disk and from the inner rim. They found that emission from the hot inner rim (being hotter than the flaring disk and thus having a higher scale height) can account for the near-IR excess in the HAEBE star AB Aur; the DDN model for AB Aur is the first disk-only model that can account for the entire SED of AB Aur.

Encouraged by our success in fitting AB Aur, we decided to investigage the applicability of the DDN model to a wider sample of HAEBE stars. We use the sample described by Meeus et al. (2001). This is a set of isolated Herbig Ae stars which was selected from a larger set of Herbig AeBe stars (Waelkens et al. 1994; Bogaert 1994; Malfait et al. 1998). To our knowledge, none of the stars (with the exception of AB Aur) shows any significant reflection nebulosity or significant extended emission on scales much larger than expected for a disk. This suggests that the SED is not contaminated by large scale dust clouds surrounding the object. The objects were further selected by their very low circumstellar extinction - typically 0.5 mag or less. Exceptions are HD 142666 (0.9 mag) and HD 150193 (1.5 mag) (and private communication, van den Ancker et al. 1998). In their paper, Meeus et al. presented combined ISO SWS/LWS spectra and literature photometry data of 14 Herbig $\mathrm{Ae} / \mathrm{Be}$ stars, and found that these objects show both striking similarities in the spectrum, and important differences.

We will attempt to interpret this variety of spectra entirely in the context of the DDN model, without additional components. This way we can find out whether an isolated disk picture is sufficient to explain the SEDs of these stars. And if we can, we will see what we can learn from the deduced parameters. The paper is organized as follows. In Sect. 2 we describe the sample. In Sect. 3 the DDN model is introduced and we detail the fitting procedure. In Sect. 4, the obtained fits are shown and discussed. Finally, in Sect. 5, we reflect on the successes and failures of the model to reproduce the SEDs of isolated Herbig stars.

\section{Sample and SEDs}

We use the sample of isolated Herbig stars as given by Meeus et al. This is a group of 13 stars which have the properties of Herbig Ae stars, but which are not located very closely to star forming regions. The original sample contains 14 stars, but we removed 51 Oph because of its uncertain nature (van den Ancker et al. 2001). The remaining SEDs are shown in Figs. 3-5. The source of the IR excess in these stars is believed to originate from a disk. The SEDs of these sources is similar, but still shows considerable variation. All stars show the characteristic bump in the spectrum around 3 micron. At a wavelength of about $5 \mu \mathrm{m}$, the SEDs $\left(\nu F_{v}\right)$ show a local 
Table 1. Measured properties.

\begin{tabular}{clccccc}
\hline \hline Group & Name & $\frac{F_{\mathrm{FIR}}}{F_{\star}}$ & $\frac{F_{\mathrm{FIR}}}{F_{\star}}$ & $\frac{F_{\mathrm{IR}}}{F_{\star}}$ & $\frac{F_{\mathrm{FIR}}}{F_{\mathrm{IR}}}$ & $\frac{F_{10 \mu \mathrm{m}}}{F_{7.7 \mathrm{~mm}}}$ \\
\hline Ia & AB Aur & 0.12 & 0.22 & 0.42 & 0.47 & 1.56 \\
Ia & HD 100546 & 0.07 & 0.39 & 0.45 & 0.15 & 6.95 \\
Ia & HD 142527 & 0.32 & 0.60 & 0.92 & 0.35 & 1.33 \\
Ia & HD 179218 & 0.10 & 0.24 & 0.35 & 0.30 & 3.85 \\
\hline Ib & HD 100453 & 0.22 & 0.29 & 0.50 & 0.43 & 2.56 \\
Ib & HD 135344 & 0.24 & 0.29 & 0.53 & 0.46 & 1.10 \\
Ib & HD 139614 & 0.09 & 0.23 & 0.32 & 0.28 & 6.53 \\
Ib & HD 169142 & 0.04 & 0.07 & 0.11 & 0.35 & 4.19 \\
\hline IIa & HD 104237 & 0.16 & 0.08 & 0.24 & 0.66 & 1.17 \\
IIa & HD 142666 & 0.14 & 0.15 & 0.29 & 0.47 & 1.82 \\
IIa & HD 144432 & 0.13 & 0.13 & 0.27 & 0.50 & 3.28 \\
IIa & HD 150193 & 0.14 & 0.13 & 0.27 & 0.52 & 1.53 \\
IIa & HD 163296 & 0.21 & 0.17 & 0.38 & 0.54 & 1.53 \\
\hline
\end{tabular}

minimum, followed by the $10 \mu \mathrm{m}$ region in which most stars show a prominent emission feature attributed to silicate. Longwards of $10 \mu \mathrm{m}$, the SEDs differ significantly. Meeus et al. found that the SEDs can be classified into two major groups, group I and group II, and they speculate that the difference between the groups can be attributed to geometry. Group I sources are objects in which the SED plotted as $v F_{v}$ stays high or even rises in the region between $20 \mu \mathrm{m}$ and about $100 \mu \mathrm{m}$. Meeus et al. attribute this strong mid-IR component to a flaring disk. Group II sources on the other hand continue to decrease longwards of $20 \mu \mathrm{m}$ (the "powerlaw component" in Meeus et al.), their spectrum in this wavelength region is more consistent with that of a geometrically thin disk. Numerically, the distinction between groups I and II can be measured by the different fractions of stellar light emitted in the near IR (NIR) and far IR (FIR) region. Table 1 shows these quantities for all stars in the sample. The stellar flux $F_{\star}$ is measured by integrating the Kurucz model fit to the optical and UV spectrum. In order to compute the emission from the disk, we subtract the Kurucz model from the SED and integrate the excess flux from $2 \mu \mathrm{m}$ to $7 \mu \mathrm{m}$ to define $F_{\text {FIR }}$ and from $7 \mu \mathrm{m}$ to infinity to define $F_{\text {FIR }}$. In the NIR, almost all stars emit between 10 and $30 \%$, with significant variation from source to source. In the FIR, group I sources reprocess typically $20-30 \%$ of the stellar radiation, while the group II sources are closer to $15 \%$. The total effect of NIR and FIR reprocessing can be seen in the quantity $F_{\mathrm{IR}} / F_{\star}$ which shows that group I sources reprocess in total around $50 \%$ of the stellar radiation, group II sources only between 20 and $30 \%$.

Meeus et al. also introduced a subdivision of the groups into the subgroups $\mathrm{a}$ and $\mathrm{b}$, which differ in the $10 \mu \mathrm{m}$ region. Sources in the "a" subgroup show strong silicate emission, while sources in the "b" subgroup have no detectable silicate emission at all. While the signal-to-noise ratio in some of the group "b" sources is limited, there is at least one case (HD 100453) where the evidence for the suppression of the $10 \mu \mathrm{m}$ feature is very strong. Interestingly, all " $\mathrm{b}$ " sources in the Meeus et al. sample belong to group I, while in group II all stars show prominent silicate emission.

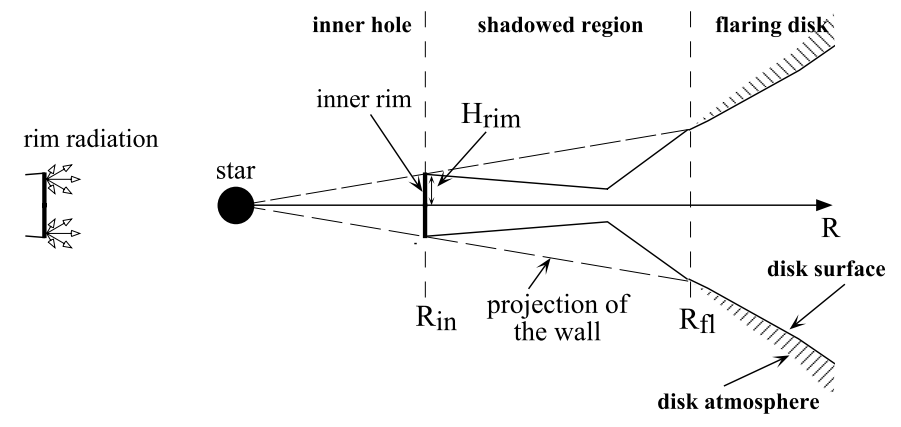

Fig. 1. Sketch of the geometrical structure of the DDN model. See text for details. After Dullemond et al. (2001).

\section{Model and fitting procedure}

\subsection{The DDN model}

The DDN disk model is based on the flaring passive disk model of Chiang \& Goldreich (1997). Improvements were made to that original model in order to conserve energy (see also Chiang et al. 2001) and to properly account for the reduction of irradiation flux due to occultation of part of the stellar surface by the disk itself. Most importantly, the DDN model includes a proper treatment of the inner rim, which is located at the dust evaporation radius. This radius is computed self-consistently. The DDN model assumes that the material inside the dust evaporation surface is optically thin to the stellar radiation. The unattenuated stellar flux impinges on the inner edge of the dusty part of the disk. Since the the inner rim exposes a vertical surface on which the stellar flux impinges perpendicularly, it gets much hotter than expected for a flaring disk at the same radius: the inner rim puffs up. The DDN model computes how much the inner rim puffs up, and how much of the disk behind it will be shadowed by this puffed-up rim (see Fig. 1). Effects of self-irradiation of the disk are included, as well as a simple treatment of radial radiative diffusion in the disk midplane, which can be important for the structure of the shadowed region. The disk model can treat low optical depths, as long as the vertical surface height is large enough that a $\tau=1$ surface can be defined for the direct stellar photons impinging under a grazing angle onto the disk. For very low mass disks, or the very outer parts of intermediate mass disks, in which even the radial optical depth at stellar wavelengths is below unity, the DDN approach breaks down.

The puffed-up inner rim is responsible for the 3-micron bump in the spectrum (Natta et al. 2001). The vertical "wall" at $1500 \mathrm{~K}$ produces the largest flux under an inclination $i$ close to edge-on. However, if the inclination is too large (close to edge-on), the observers sees the inner regions of the disk through the outer disk, and the inner rim as well as the central star will be obscured. None of the stars in the Meeus sample have large extinction towards the star, so we can rule out this extreme case. In the DDN model, the 3 micron flux also vanishes for exact face-on inclination $(i=0)$. This is a geometrical effect and is due to the fact that the emissions originates from the surface of a cylinder. The projected surface of this cylinder vanishes for low inclinations. In reality, the wall will likely be curved, weakening this effect. 


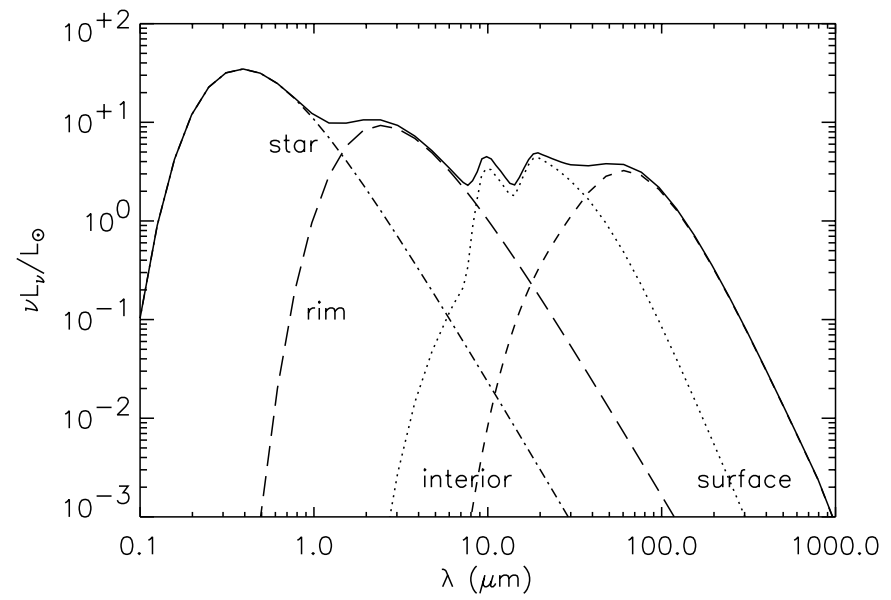

Fig. 2. An example of an SED of a Herbig Ae star, as computed by the DDN model. The spectrum as shown here is decomposed in its four main components: star, inner rim, disk surface and disk interior. The star is approximated with a blackbody curve.

The disk directly behind the inner wall is shadowed by the wall itself. It does not receive direct stellar radiation and will therefore not radiate significantly. In the SED, the contribution of this region is negligible. At larger radii, the flaring disk reappears from the shadow, and emits as the usual flaring passive irradiated disk, producing mid- and far infrared emission. If the disk is rather massive, it will be optically thick at most wavelengths in the infrared and produce a rather flat spectrum (in $v F_{v}$ ). The flat spectrum which is a typical sign of flaring disks results from the sum of two equally strong components: an optically thin component in the mid-IR with emission features (the disk's surface layer), and an optically thick component at far-IR wavelengths (the disk's interior).

The spectrum of the disk will therefore consist of three major components: the 3 micron emission from the inner rim, the mid-IR emission from the surface layer, and the far-IR emission from the disk's interior (see Fig. 2). The basic shape of the SED can be easily understood in terms of energy conservation. The total flux in the infrared excess compared to the stellar flux is determined by the covering fraction of the disk with respect to the central star. This covering fraction is equal to the ratio $H_{\mathrm{s}} / R$ (surface height over radius) at the outer edge of the disk, where the disk geometric thickness is maximum. In general one can say that the covering fraction increases for larger mass and/or larger outer radius, and is only weakly dependent on other parameters of the disk. The other parameters determine how this fixed amount of IR excess is divided over the three components. For instance, a large inner rim height will put a lot of flux in the 3-micron bump at the cost of the mid- and far-IR. For more details on how the disk parameters affect the SED we refer to the DDN paper.

\subsection{The dust opacities}

In the disks we are studying, the continuum opacity is entirely dominated by the dust. We use dust grains of $0.1 \mathrm{mi}-$ cron in size, belonging to one of the following different dust species: astronomical silicate (Draine \& Lee 1984), carbon
Table 2. The parameters entering into the DDN model.

\begin{tabular}{ll}
\hline \hline Parameter & observed or fitted? \\
\hline$L_{\star}$ & from observations (Meeus et al.) \\
$M_{\star}$ & from observations (Meeus et al.) \\
$T_{\text {star }}$ & from observations (Meeus et al.) \\
$T_{\text {rim }}$ & fixed at 1500 K \\
Dust & mostly fixed composition: $0.1 \mu \mathrm{m}$ grains, 5 \\
& or 10\% carbon, the rest silicate. $\beta$ slope of \\
& silicate opacity either one or two, fitted. \\
$M_{\text {disk }}$ & fitted (minimum value if not unique) \\
$p$ & fitted \\
$r_{\text {out }}$ & fitted \\
$\chi$ & fitted for group Ib sources, otherwise very \\
$i$ & close to selfconsistent value. \\
& inclination from observations when avail- \\
& able, otherwise fitted \\
\hline
\end{tabular}

(Laor \& Draine 1993), and forsterite (Servoin \& Piriou 1973; Scott \& Duley 1996). These three species can be mixed in any composition, but they are assumed to be thermally coupled, i.e. we assume a single temperature for all dust species. In most cases we assume a standard mixture of dust: $90 \%$ silicate and $10 \%$ carbon. We do not attempt to fit in detail all the spectral features in the SED, in particular we do not include PAH's even though they are clearly present in some objetcs. However, in one case (HD 100546) we include crystalline forsterite in order to match the extremely strong contribution of these features in this source. In general, however, we focus on fitting the SED which is only weakly dependent on the actual dust composition. The exception is the (sub-)mm range of the spectrum: this part is critically dependent on the long-wavelength behavior of the silicate opacity. There is long standing debate about the slope of the opacity in this range. The opacity of Draine \& Lee goes as $\kappa_{\lambda} \propto \lambda^{-2}$ while from observations of the interstellar medium it follows that $\kappa_{\lambda} \propto \lambda^{-1}$ (Beckwith et al. 2000). Many of the sources in our sample (with the notable exception of $\mathrm{AB}$ Aurigae) can be fitted better with an opacity that goes as $\kappa_{\lambda} \propto \lambda^{-1}$ at long wavelengths. So we add an adapted silicate opacity that is identical to the Draine \& Lee opacity for $\lambda<100 \mu \mathrm{m}$ and goes as $\kappa_{\lambda} \propto \lambda^{-1}$ for $\lambda>100 \mu \mathrm{m}$. By gluing the two opacity regimes smoothly together at $\lambda=100 \mu \mathrm{m}$, the long wavelength opacity in fact turns out to have exactly the same normalization as the opacity proposed by Beckwith et al. (2000).

\subsection{Fitting procedure}

In order to fit the SEDs of the stars, we run the DDN model and change parameters until we find a fit to the observed spectrum. To minimize the chance of missing important regions of parameter space, and to maximize our understanding of the fitted solutions, we employ a specially developed a widget interface that allows one to do on-the-spot fitting of the model to the observed SED. 
Table 3. Stellar properties and fit parameters. In the column of $\chi$ the numbers which are bracketed are results of the self-consistent determination of this parameter. If not bracketed, it is fitted by hand.

\begin{tabular}{|c|c|c|c|c|c|c|c|c|c|c|c|c|c|c|c|}
\hline Group & Name & SpType & $\begin{array}{l}M_{\star} \\
M_{\odot}\end{array}$ & $\begin{array}{l}L_{\star} \\
L_{\odot}\end{array}$ & $\begin{array}{r}T_{\text {eff }} \\
\mathrm{K}\end{array}$ & $\begin{array}{r}d \\
\mathrm{pc}\end{array}$ & $\begin{array}{r}M_{\text {disk }} \\
M_{\odot}\end{array}$ & $p$ & $x$ & $\begin{array}{l}r_{\text {out }} \\
\mathrm{AU}\end{array}$ & $i$ & $f_{\mathrm{sil}}$ & $f_{\text {sil1 }}$ & $f_{\text {fors }}$ & $f_{\mathrm{c}}$ \\
\hline Ia & $\mathrm{AB}$ Aur & B9/A0Ve & 2.50 & 47 & 9750 & 144 & 0.100 & -2.0 & (5.5) & 400 & 65 & 95 & 0 & 0 & 5 \\
\hline Ia & HD 100546 & B9Ve & 2.50 & 36 & 11000 & 103 & 0.005 & 0.0 & 1.2 & 400 & 51 & 0 & 70 & 17 & 11 \\
\hline Ia & HD 100546 & B9Ve & 2.50 & 36 & 11000 & 103 & 0.020 & 2.0 & $(0.0)$ & 43 & 30 & 47 & 0 & 47 & 5 \\
\hline Ia & HD 142527 & F7IIIe & 2.50 & 31 & 6250 & 200 & 0.010 & -1.0 & (4.3) & 200 & 75 & 0 & 90 & 0 & 10 \\
\hline Ia & HD 179218 & B9e & 2.70 & 80 & 10000 & 240 & 0.010 & 0.8 & (3.4) & 30 & 20 & 80 & 0 & 0 & 20 \\
\hline $\mathrm{Ib}$ & HD 100453 & $\mathrm{~A} 9 \mathrm{Ve}$ & 1.70 & 9 & 7500 & 114 & 0.010 & -0.5 & 6.0 & 600 & 65 & 90 & 0 & 0 & 10 \\
\hline $\mathrm{Ib}$ & HD 100453 & A9Ve & 1.70 & 9 & 7500 & 114 & 2.000 & -1.0 & (5.3) & 300 & 75 & 0 & 0 & 0 & 10 \\
\hline $\mathrm{Ib}$ & HD 135344 & $\mathrm{~F} 4 \mathrm{Ve}$ & 1.30 & 3 & 6750 & 84 & 0.010 & -0.8 & 8.0 & 800 & 60 & 0 & 90 & 0 & 10 \\
\hline $\mathrm{Ib}$ & HD 139614 & A7Ve & 1.80 & 12 & 8000 & 157 & 0.010 & -0.1 & 5.0 & 60 & 20 & 0 & 94 & 0 & 5 \\
\hline $\mathrm{Ib}$ & HD 169142 & $\mathrm{~A} 5 \mathrm{Ve}$ & 2.50 & 32 & 10500 & 145 & 0.100 & -2.0 & 7.0 & 100 & 8 & 0 & 90 & 0 & 10 \\
\hline IIa & HD 104237 & A4Ve & 2.60 & 40 & 10500 & 116 & 0.060 & 0.0 & (4.8) & 10 & 55 & 0 & 90 & 0 & 10 \\
\hline IIa & HD 142666 & $\mathrm{~A} 8 \mathrm{Ve}$ & 1.80 & 11 & 8500 & 116 & 0.030 & 0.5 & (4.3) & 10 & 55 & 0 & 90 & 0 & 10 \\
\hline IIa & HD 144432 & A9Ve & 2.20 & 32 & 8000 & 200 & 0.200 & 0.5 & (4.8) & 10 & 45 & 0 & 90 & 0 & 10 \\
\hline IIa & HD 150193 & $\mathrm{~A} 1 \mathrm{Ve}$ & 2.50 & 40 & 10000 & 150 & 0.010 & 0.5 & (4.3) & 8 & 45 & 0 & 90 & 0 & 10 \\
\hline IIa & HD 163296 & $\mathrm{~A} 3 \mathrm{Ve}$ & 2.40 & 30 & 10500 & 122 & 0.050 & -0.2 & (4.3) & 50 & 65 & 0 & 90 & 0 & 10 \\
\hline
\end{tabular}

In our fitting procedure we do not vary all the parameters of the DDN model. Whenever possible, we used observations to constrain specific parameters. In particular, the stellar properties were inferred from observed parallax, magnitude and spectral type. There are some uncertainties in the inferred values of, in particular, the mass and the luminosity of the star. We have taken the luminosities provided by Meeus et al., and the masses given by van den Ancker et al. (1997). The uncertainties in the luminosity stem from the error in the Hipparcos distances and from uncertainties regarding the dereddening which is crucial for the determination of the bolometric luminosity of hot stars. The uncertainties in the luminosities are typically up to $50 \%$. The stellar masses were obtained by placing the stars into an HR diagram with overlayed pre-main-sequence evolutionary tracks. The uncertainties in the masses are relatively small. Van den Ancker et al. quote 5-10\% statistical error.

The main disk parameters that are varied in order to fit the SED are the disk mass $M_{\text {disk }}$, the slope $p$ of the powerlaw describing the radial dependence of the surface density, the outer disk radius $r_{\text {out }}$, the inclination $i$ and, in some cases, the height of the inner rim in units of the local pressure scale height $\chi_{\text {rim }} \equiv\left(H_{\mathrm{s}} / H_{\mathrm{p}}\right)_{\text {rim. }}$. The latter parameter is usually computed self-consistently, but occasionally is fine-tune by hand. For some sources (AB Aur, HD 100546, HD 163296) the outer radius could be assumed to be the radius measured by continuum interferometry at $2.6 \mathrm{~mm}$ (Mannings \& Sargent 1997). However, we consider these radii to be inaccurate, since they only tell at which radius the signal-per-synthesized-beam drops below the noise. We therefore kept the outer radius a free fitting parameter. The observed radii do, however, provide a lower limit to the disk radius.

When we try to fit a particular SED, we put most of the weight on the IR region of the spectrum. This is the region where most of the reprocessed stellar energy emerges, and therefore this part of the spectrum is most strongly constrained by the model. This approach sometimes leads to underestimating the submm fluxes. While not completely satisfactory, the deviating submm fluxes are energetically irrelevant. The large uncertainties in the $\mathrm{mm}$ and radio dust opacities also indicate that these points provide less solid constraints to disk models. Additional flux in the submm region can be produced by introducing an addtional component of large dust grains in the midplane, as has been done by many authors (e.g. Natta et al. 2001; Bouwman et al. 2000). This is basically equivalent to changing the wavelengths dependence of the opacity in the region.

Table 2 summarizes the input parameters of a DDN model.

\section{Results}

\subsection{The group la sources}

The group Ia sources show SEDs which are reminiscent of the SEDs of T Tauri stars. The SED is flat or even rising from 1 to about $60 \mu \mathrm{m}$, a clear sign for a flaring disk geometry. Typically about $50 \%$ of the stellar radiation is reprocessed by the disk and emitted in the infrared, approximately equally distributed between the $3 \mu \mathrm{m}$ bump between 2 and $7 \mu \mathrm{m}$, and the rest of the infrared spectrum. AB Aur is the prototype of this group, and the best studied Herbig Ae star. Apart from AB Aur the Meeus et al. sample contains three more stars in the group Ia: HD 100546, HD 142527, and HD 179218. The SEDs and our fits for these stars are shown in Fig. 3, and the fit parameters are given in Table 3 . It is immediately visible from the plots, that, while these sources are similar to AB Aur, they also show pronounced differences.

\subsubsection{AB Aurigae as a prototype}

In the DDN paper the standard source was AB Aurigae, which is the best studied Herbig Ae star. We show our fit to the SED in Fig. 3 and list the parameters used in Table 3. The parameters slightly differ from those used by DDN. The current values 


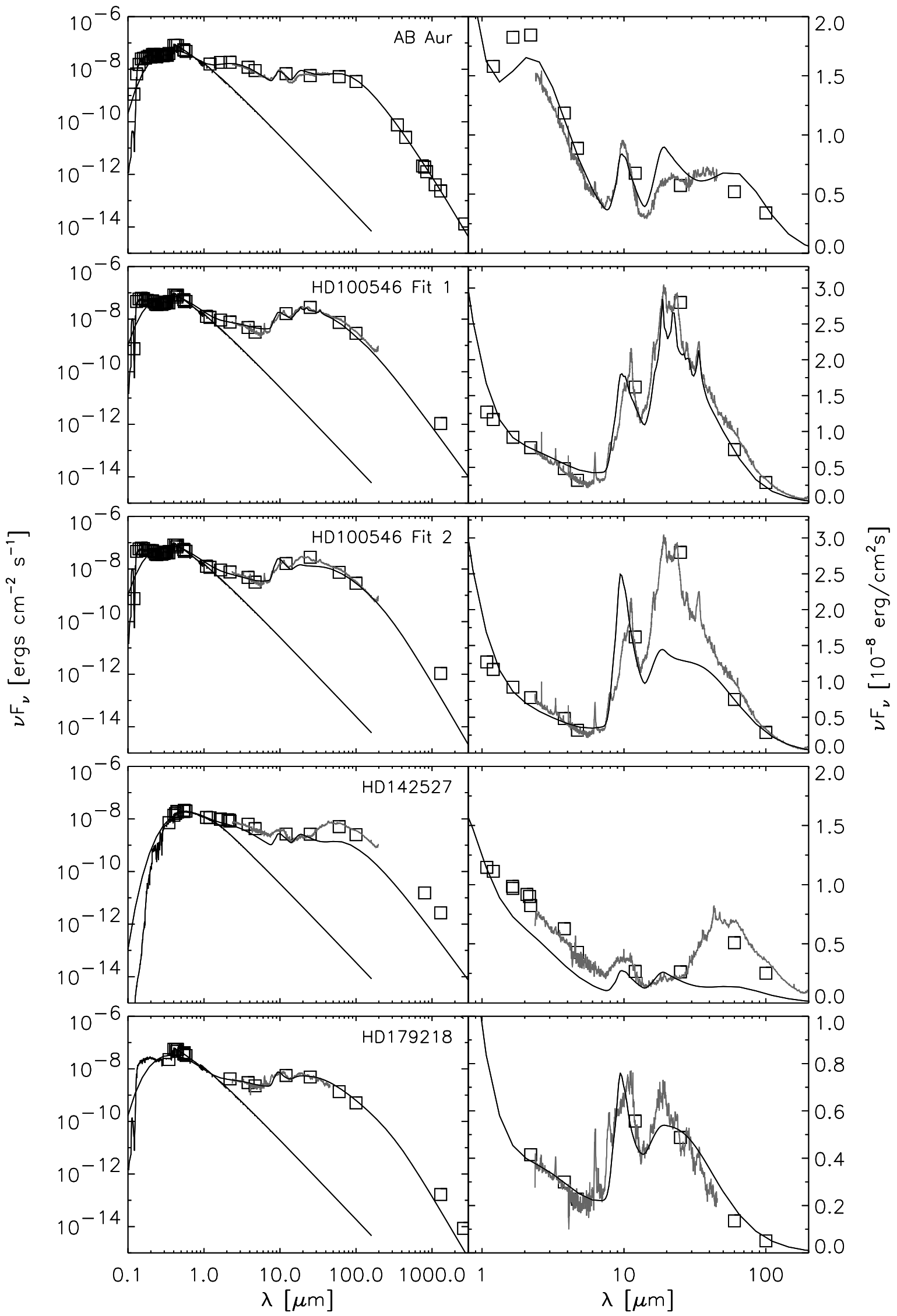

Fig. 3. The group Ia sources. The left column shows the full SED $v F_{v}$ of the star on a log-log scale together with a Kurucz model for the star and a model fit for the disk. The right column shows the region from 1 to 100 micron with a linear $y$-axis in order to allow for a better comparison between model and observations. The grey line show the ISO spectrum, the squares are photometry points. 


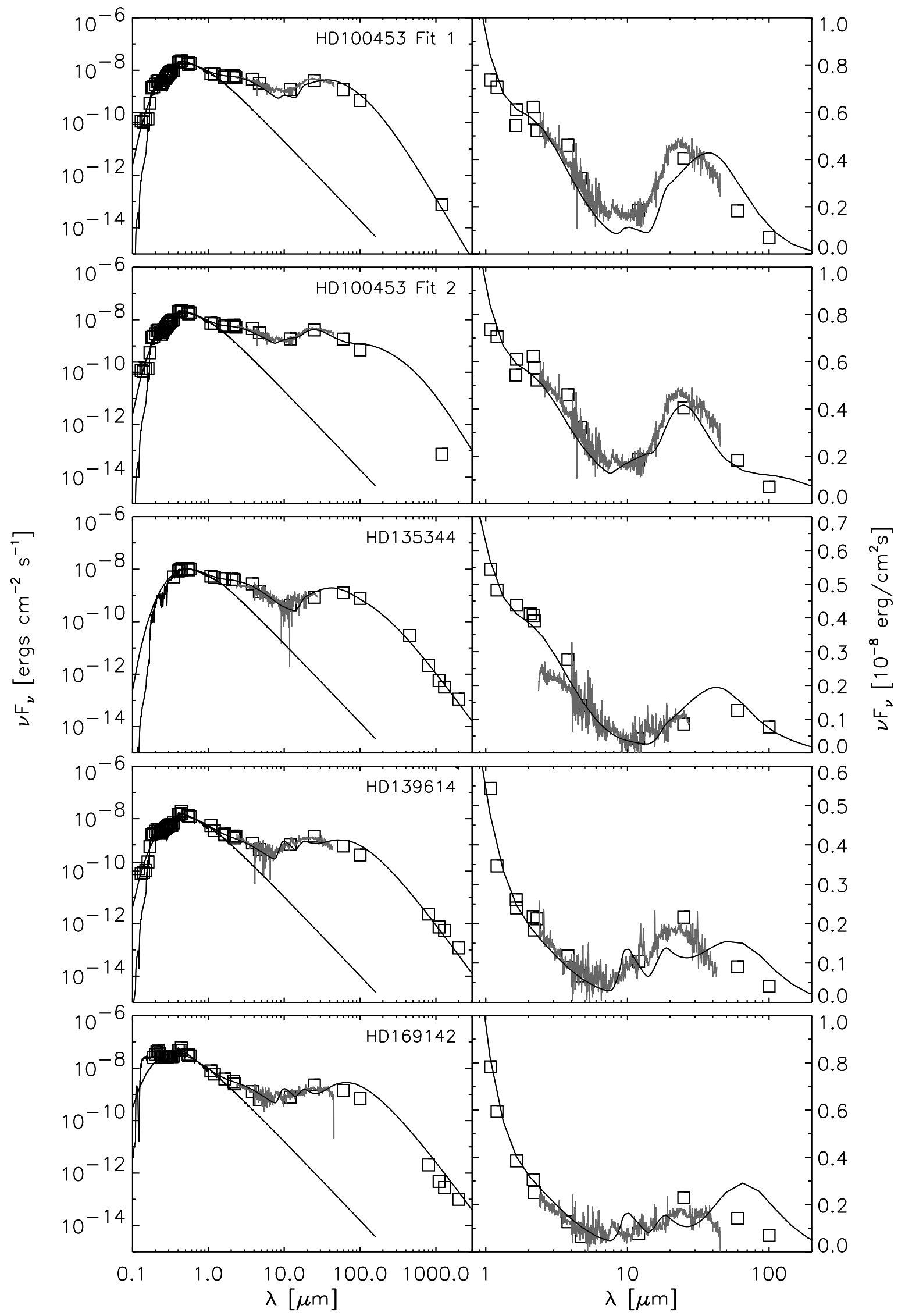

Fig. 4. The group Ib sources. See caption of Fig. 3. 


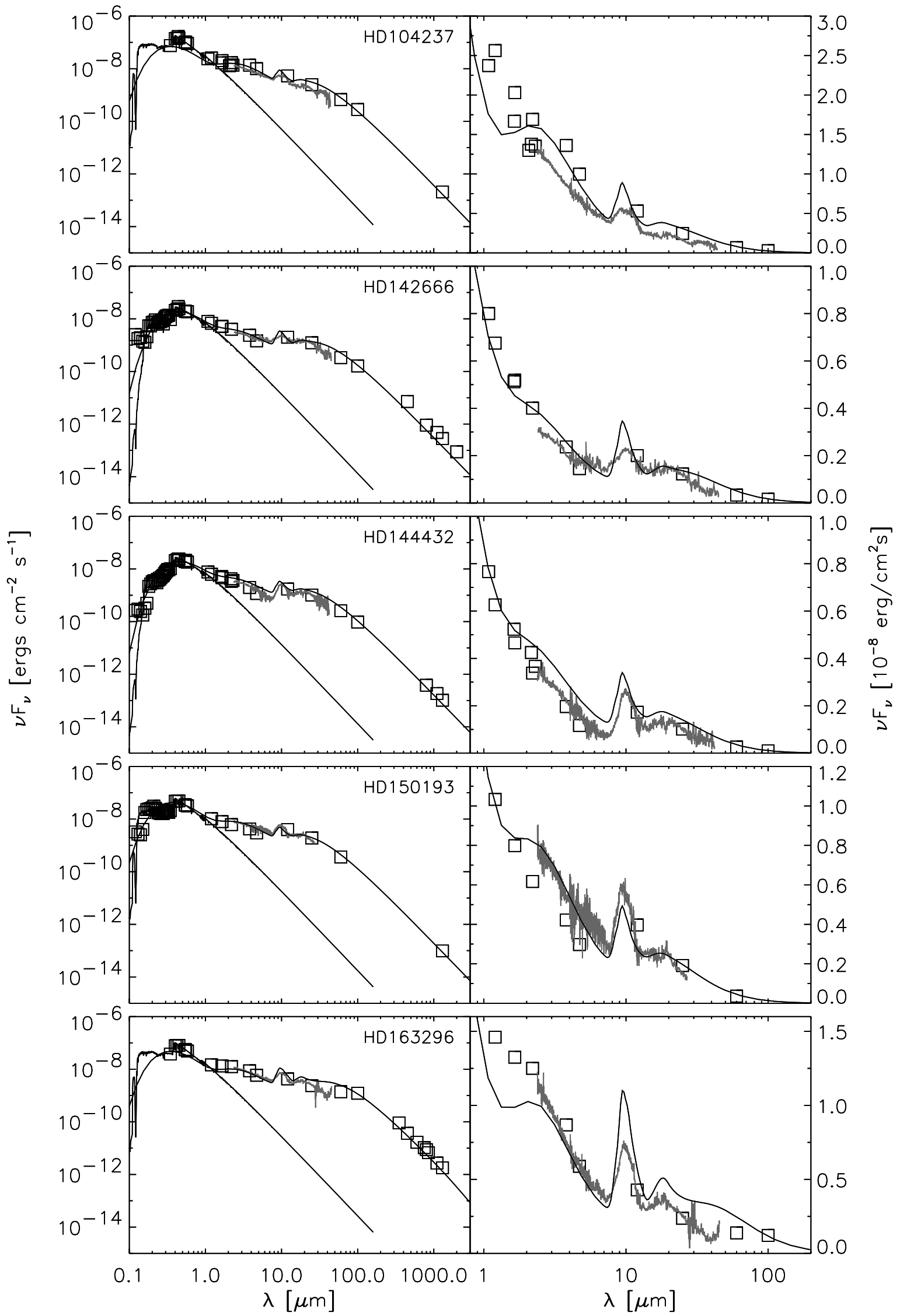

Fig. 5. The group IIa sources. See also caption of Fig. 3. 
are more consistent with the measured size of the disk and the inclination inferred from mm observations (e.g. Mannings \& Sargent 1997). The model fits AB Aur very well. The main difficulties are a small under-prediction at the onset of the excess and an incorrect ratio of the $10 \mu \mathrm{m}$ and $20 \mu \mathrm{m}$ amorphous silicate features. The model parameters show what is typically needed to fit a group I source: a decreasing surface density and an outer radius of several hundred AU. The submm slope is steep, so the normal Draine \& Lee silicate opacities can be used to fit this source.

\subsubsection{HD 100546}

HD 100546 is a very interesting group Ia source. It shows an extremely steep rise starting at $7 \mu \mathrm{m}$. The spectrum is dominated by very strong emission features of crystalline silicates (Malfait et al. 1997). In order to reproduce the crystalline features, we introduced crystalline Forsterite as an additional dust component for this star.

In $\mathrm{AB}$ Aur about $50 \%$ of the reprocessed radiation is located in the rim component and $50 \%$ in the flaring component. In HD 100546 these corresponding numbers are $30 \%$ and $70 \%$ (Bouwman et al. 2001). With the self-consistently computed inner rim height, the rim component becomes much too luminous and the flaring component falls far below the observed huge bump between 7 and $100 \mu \mathrm{m}$. The only solution which produced a decent fit was to enforce a lower rim with $\chi_{\text {rim }}=1.2$ instead of the usual 4-5. This reduces the rim component to the observed values. The flaring part of the disk then receives more radiation and becomes more luminous, illustrating the trading of energy between components in a passive disk model. With a radially decreasing surface density, the scale height of the disk in the flaring region was still too small to reproduce the flux in the $20-40 \mu \mathrm{m}$ region, but a satisfactory result was achieved with a constant surface density. In practice this means that most of the disk material is located in the outer regions of the disk. The region between the rim and about $10 \mathrm{AU}$ is almost empty. This is therefore similar to the gap proposed by Bouwman et al. (2002). In order to produce the strong crystalline emission features, we need a ratio of crystalline to amorphous material of $1: 3$, consistent with the cool component in the Bouwman et al. model.

A low surface density in the inner regions also provides a physical explanation for the low rim height. To illustrate this, we have also computed an alternative model, in which we assume the surface density to increase with distance from the star. We show this fit in the third row of Fig. 3. An increasing surface density leads in practice to even less mass in the inner disk regions which will then become marginally optically thin. If we use $p=2$, we can reproduce the low height of the inner rim in a selfconsistent way. However, the overall fit of this model is not quite as good as the first model. In particular the ratio between 10 and 20 micron region becomes incorrect.

\subsubsection{HD 142527}

The fit to HD 142527 is clearly much less satisfactory, but this is not surprising. The numbers listed in Table 1 show that the IR excess emission contains almost as much flux as the star itself, a property which is impossible to fit with a passive reprocessing disk model. Both the NIR and the FIR components are larger than in any other star in the sample. Reproducing the emission in the 3 micron region would require a high inner rim, with approximately 7 pressure scale heights, unrealistic for a hydrostatic disk. In addition, a high rim casts a long shadow on the flaring disk region, so that the model necessarily predicts a relatively weak far-IR spectrum, and gives hardly any rise at $20 \mu \mathrm{m}$. If indeed the near-IR flux originates from the inner rim of the disk, then the huge bump in the flux from the object around $60 \mu \mathrm{m}$ probably does not originate from a disk, but from additional material in the same line of sight. Another possibility is that we see this star partially through the disk, and that therefore the stellar flux is greatly underestimated. In fact, we have found several different estimates for the luminosity of HD 142527, including one which is a factor of two higher (van den Ancker et al. 1997). However, even with this higher luminosity, no satisfactory fit could be obtained. It is possible that within the beam of both photometry and ISO observations, there is another source of radiation peaking at $60 \mu \mathrm{m}$. However, the source is likely associated with HD 142527 since the emission shows significant crystalline silicate features implying processed material, as well as crystalline $\mathrm{H}_{2} \mathrm{O}$ ice.

\subsubsection{HD 179218}

Somewhat similar to HD 100546, HD 179218 has a relatively low near-IR emission, and a strong far-IR component. Again we need an extreme powerlaw for the surface density in this star: $p=2$. So also for this star, most of the mass is located in the outer regions of the disk. The resulting low rim height reproduces the low near-IR flux. Most of the reprocessing takes place in the flaring part of the disk, which reproduces the midand far-IR flux. The width of the SED is much smaller than in $\mathrm{AB}$ Aur, which is consistent with the small disk size we derived.

\subsection{The group lb sources}

The overall shape of spectra of the group Ib sources are similar to the ones from group Ia, but very conpiciously the silicate feature is absent in these spectra. There are two main possible explanations for the absence of emission features. Either the dust grains are all large ( $a=3 \mu \mathrm{m}$ or larger), so that they will be opaque at $10 \mu \mathrm{m}$ and not produce a feature. Or the geometry of the disk is such that no silicate grains of the right temperature are present. DDN noted that this can be achieved with a purely geometrical effect: If the inner rim of the disk is high enough (about 5-7 pressure scale heights), then the region of the disk which normally produces the $10 \mu \mathrm{m}$ silicate emission feature is located entirely in the shadow, and the corresponding component is missing from the spectrum. The self-consistently computed rim height is usually not large enough to achieve this. 
We therefore tried to fit the SEDs of these sources by treating $\chi_{\text {rim }} \equiv\left(H_{\mathrm{s}} / H_{\mathrm{p}}\right)_{\text {rim }}$ as a free parameter. Figure 4 shows our attempts to do so. Similarly, a gap in this $10 \mu \mathrm{m}$-feature producing region could also have this effect, but the possibility for a physical gap is currently not included in the DDN model.

\subsubsection{HD 100453}

The ISO spectrum of HD 100453 has the best signal-to-noise ratio of all group $\mathrm{Ib}$ sources, and therefore provides the strongest constraints on the absence of solid-state emission features (Meeus et al. 2002). A very weak feature may be hidden in the noise of the spectra. In fact, van Boeckel et al. (2002, in preparation) show with high quality ground-based data that even HD 100453 possesses a very weak feature.

In order to fit the spectrum of HD 100453, we used a rim height of 6 pressure scale heights, as opposed to the computed 3.3. This height is sufficient to shadow the $10 \mu \mathrm{m}$ emitting region. However, the large shadow also decreases the emission from the disk interior at these radii, and the model falls short between 8 and $30 \mu \mathrm{m}$. Our best model which is shown in Fig. 4 shows a steep rise, but at longer wavelengths than observed. We have not been able to find a set of parameters which at the same time suppresses the silicate feature and fits the steep rise in the spectrum starting at $12 \mu \mathrm{m}$. At mm wavelength, the fit is very good again, running through the $30 \mathrm{mJy}$ measurement ( $\mathrm{S}$. Wolf, priv. communication).

The other possibility to produce a spectrum without a silicate emission feature is to use large grains. It has been shown by Meeus et al. (2002) that a depletion of hot small silicate grains by a factor 300 compared to AB Aur would make the silicate feature disappear. In order to demonstrate this possibility, we have made a second fit to the SED of HD 100453 , using only large $(3.3 \mu \mathrm{m})$ silicate grains. This fit is shown in the second row of Fig. 4. Here we can fit the SED very satisfactory. However, since the opacity per unit mass is reduced significantly, we need an unphysically large disk mass $\left(2 M_{\odot}\right)$ in order to keep the required scale height of the disk, in particular in the rim region. The obvious solution to this problem would be to use a dust composition which is not well mixed over the entire disk. In particular, if the opacity of the surface layer is provided by non-silicate dust (e.g. small iron grains, carbon grains or even large molecules like PAHs), the absence of the silicate feature could be achieved by either coagulating the silicate grains to large ones, or by settling the small silicates so that they do not receive direct stellar radiation.

\subsubsection{HD 135344}

The fit for HD 135344 is satisfactory from about 10 micron all the way down into the mm region. The inner rim height had to be increased to $\chi_{\text {rim }}=6$, and we need a relatively large disk (800 AU) in order to get the rather broad spectrum observed. In the region below $10 \mu \mathrm{m}$, a significant difference between the photometry points and the ISO spectrum can be observed. The model fit is good for the SWS spectrum, but clearly stays below the photometry points. The reason for this observational discrepancy is not known. Maybe the source is variable in the NIR. Monitoring at these wavelengths would therefore be useful.

\subsubsection{HD 139614}

The model fit for HD 139614 suffers from the same problems as the fit for HD 100453. We can fit the NIR emission and the absence of the silicate feature with an artificially high inner rim, but the price for this is again too little flux in the $10-40 \mu \mathrm{m}$ region. Moreover, HD 139614 has a relatively low near-IR flux, which can only be reconciled with the high inner rim when the inclination is very small (face-on). See HD 169142 below for a discussion on this problem, which is even more accute in that source. A reasonably over-all fit can be found (see Table 3), but a still relatively strong silicate feature appears.

\subsubsection{HD 169142}

The SED of the star HD 169142 is characterized by a low infrared flux compared to the other members of its group. In terms of an optically thick disk model this can only be interpreted in two ways: either we see the disk at an inclination in which it happens to emit only very little flux, or the disk itself has a very low reprocessing capability. In the purely absorbingreemitting disk model of DDN, this means that the disk covers a relatively small fraction of the sky as seen by the star, i.e. the disk is rather flat. This "covering fraction", however, is a very weak function of the disk parameters. Only by strongly reducing the outer radius of the disk, and suppressing the height of the inner rim, one can achieve this naturally. Chiang et al. (2001) suffered from a similar problem. They suggested that dust settling has lowered the $\tau=1$ surface of the disk, and thus the covering fraction of the disk is reduced.

We fitted a DDN disk with relatively low optical depth at large radii (i.e. small mass, and steep $\Sigma$-powerlaw), and with a very small inclination. The small inclination suppresses the near-infrared flux because the emission from the inner rim is emitted as a cylinder emitting from the inside: when seen at $i=0$, the flux is zero. It is unclear how realistic this is because of the uncertain geometrical shape of the inner disk rim. Furthermore, the best fit still shows a weak 10 micron silicate feature which seems to be absent in the spectrum. Higher $\mathrm{S} / \mathrm{N}$ ratio observations would be very useful to confirm the presence or absence of a silicate feature in this star.

\subsection{The group lla sources}

The overall spectrum of the group IIa sources show similarities and differences with the group Ia sources. The initial onset of the IR excess is still at $3 \mu \mathrm{m}$, consistent with an inner disk boundary at the evaporation temperature of the dust. Also, the amount of energy reprocessed in this component is similar. However, the group II sources don't show an additional rise of the spectrum beyond $20 \mu \mathrm{m}$, so the flaring component is strongly suppressed. In the frame of the DDN model this implies a truncation of the disk to very small sizes.

However, since the submm fluxes of theses sources are comparable to the fluxes measured for group Ia sources, the disk mass in both cases has to be similar. In view of the very small disk size, the disk is therefore completely optically thick up to wavelengths of about 100 to sometimes 1000 micron. 
The only model for which the disk becomes optically thin at 1000 micron is the model for HD 150193. At first sight, this seems to be inconsistent with the steep slope of the spectra of group II sources (with significantly less power in the far-IR than in the mid-IR), since optically thick reprocessing disks tend to produce mid- and far-IR fluxes of equal strength in $v F_{v}$. However, due to the very small radius of the disk, the flaring component is weak in comparison to the inner rim component. The inner rim component will significantly contribute to the mid-IR flux, but not to the far-IR flux. This naturally produces the steep slope observed. The truncation of the disk is such, that the disk starts to flare just before the truncation radius. The small flaring is mainly required in order to produce a silicate emission feature which is present in all known group II sources. Meeus et al speculated that this may be a selection effect: group II sources are relatively weak IR emitters, and therefore only the brightest have been selected for the ISO study.

An alternative to a truncated disk is a collapsed disk outside of about $20 \mathrm{AU}$, so that the material outside this radius is not directly exposed to the stellar light, and will only be heated indirectly by the infrared radiation of the inner part of the disk. In the context of the simplified model of DDN such a disk cannot be modeled properly. Detailed 2-D radiative transfer is required, which is outside the scope of this paper. Clearly, such a model could help solve the difficulties with fitting some of the far-IR an submm fluxes by supplying extra flux at these long wavelengths.

\subsubsection{HD 104237}

This object is quite well fitted with a small disk. There is some freedom in choosing the outer radius and the power law slope of the surface density, as long as the outer radius is of order $10 \mathrm{AU}$ to $20 \mathrm{AU}$. For an outer radius smaller than $3 \mathrm{AU}$ the entire disk would be in the shadow of the inner rim, and we exclude this possibility. In the near-IR the photometry points are considerably above the ISO points. Out fits runs between the two measurements. A similar problem happens at around 20-100 microns. Our fit matches the photometry points better than the ISO fluxes, and runs straight through the 1.3 millimeter point.

\subsubsection{HD 142666}

Also for this star, a compact disk give a very good fit throughout the IR region of the spectrum. The model again underpredicts the mm fluxes, so some cold dust, perhaps in a collapsed outer disk may be needed to explain the entire spectrum.

\subsubsection{HD 144432}

A fully self-consistent (including $\chi_{\text {rim }}$ ) model fit can be obtained for a small disk. This time, the mm points do not cause a problem for the self-consistent determination of $\chi_{\text {rim }}$.

\subsubsection{HD 150193}

Also for HD 150193 a fully self-consistent (including $\chi_{\text {rim }}$ ) model fit can be obtained for a small disk.

\subsubsection{HD 163296}

The spectrum of HD 163296 seems to be intermediate between group I and group II sources in several ways. The decrease in $v F_{v}$ only starts at 100 micron, which is at longer wavelengths than for the other group II sources. Initially the disk SED starts to decrease at about 30 micron like the other sources, but then recovers until 100 micron. Such an extra "bump" cannot be fitted easily with a continuous flaring disk. Our fit is therefore a compromise: It tries to fit the 100 micron point and consequently overpredicts the flux in the 60 micron region. The outer radius of the model fit is given by $50 \mathrm{AU}$, also intermediate between the typical values for group Ia and group IIa. This size is still a factor of two smaller than the continuum measurement of $100 \mathrm{AU}$ (Mannings \& Sargent 1997), while the CO data even gives $310 \mathrm{AU}$.

\section{Discussion}

The attempt to fit the spectra of isolated Herbig Ae stars with a physical disk model requires different typical sets of parameters for the different disks. Overall, the fits are reasonable for group Ia and even better for IIa. Group Ib sources require large grains to hide the silicate feature effectively without affecting the cold parts of the disk. What remains to be seen is if the fit parameters are physically realistic and reasonable.

\subsection{Group la}

The majority of the goup I sources can be well fitted with large (few hundred AU) disks. The surface density distribution needed for these sources varies greatly. For AB Aur, a powerlaw $p=-1.5$ works. This surface density slope is consistent with the structure of the solar nebula as derived from the planet masses, and also a reasonable result for accretion disks (Hayashi 1981). On the other hand, for HD 100546 and HD 179218 we find that a surface density increasing with distance from the star is required. This can be understood as a largely cleared-out inner region with still copious amounts of material present in the outer regions of the disk. This picture is quite different from a normal accretion disk which leads to a decreasing surface density with distance. In disks with gaps (possibly created by planets), accretion accross the gap will be severely decreased (Artymowicz \& Lubow 1996) and the supply of material which feeds the inner disk dies out. Since the viscous timescales in the inner disk are short, the entire part inside the gap will be emptied quickly, which may produce a structure consistent with the density distribution required for our fits. We suggest that the inner disks of these two sources are starved, and have lost much of their initial mass content by accretion. The current accretion rate in these sources should be extremely low. 


\subsection{Group Ib}

The attempt to explain the absence of the silicate feature in the group Ib sources by rim shadowing seems to have failed. First of all, the required rim height is considerably higher than typical hydrostatic rims can provide. The height of the rim is defined as the highest radial ray through the inner rim which still becomes optically thick for stellar radiation. In hydrostatic equilibrium, the vertical density structure in the disk is given by a Gaussian distribution. For normal surface densities, the surface height in the rim is typically between 4 and 5 pressure scale heights, clearly in the exponential range of the Gaussian density distribution. In order to change the densities enough for a $30 \%$ increase of the rim height, the surface density in this region would have to increase by a factor of 10 . Therefore, a hydrostatic rim height of 6 or even 7 pressure scale heights seems unrealistic. It may be possible to have hydrodynamic fluctuations sling matter towards higher elevations above the midplane, a phenomenon which might be related to the variability observed in UX Orionis stars. However, such a scenario would predict a variable shadowing effect rather than a complete blocking of the stellar light. Due to the lack of spectra taken at different epochs, such a variability cannot be excluded at the present time.

Another possible way to suppress the 10 micron feature by a geometrical effect is by "frustrated flaring". In the DDN picture, the disk rises out of the shadow as soon as this is possible, assuming the shape of a normal Chiang \& Goldreich flaring disk in the outer regions. However, it cannot be ruled out that the flaring of the disk starts further out in the disk. It is then possible that the 10 micron emitting region remains in the shadow of a normal rim. As the disk reappears from the shadow at larger radii, its flaring will be stronger than usual to compensate for the lost flux. This would produce a larger-than-normal bump in the spectrum, of which the SEDs of HD 100546, and HD 179218 may be examples. In a recent study, Calvet et al. (2002) have used a similar model to explain the SED of the T Tauri star TW-Hydra. They propose that a planet clears out part of the inner region, so that the disk can only start to flare further away from the star.

Finally, the best solution to the "missing silicate mystery" may be that the silicate grains have coagulated to large grains or settled to the disk interior, or both. The opacity of the disk surface must in this case be provided by other small grains or large molecules which will neither settle nor coagulate, but still provide optical and UV opacity. The fact that all group IIa sources show PAH emission may point into this direction.

\subsection{Group Ila}

The group II sources can be fit really well with very compact disks with outer radii as small as 10 AU. Since the sub-mm measurements of these disks are not very different from the group Ia sources, the disk mass must be similar. This combination of high mass and small size leads to very high surface densities, and to disks which are optically thick up to $\mathrm{mm}$ wavelengths. Unfortunately, sizes for most of these disk are unknown, with the only exception of HD 163296, which is the least compact disk according to our fits. The observed size of $100 \mathrm{AU}$ is a factor of $\mathrm{w}$ larger that our best fit model.

The compact size seems to be inconsistent with disk sizes typically measured around young stars. Our own solar system, the diameters of some Vega-like debris disks (Aumann et al. 1984) and the sizes of proplyds observed in the Orion starforming region (O’Dell 1998) all point to disk sizes which are at least $50 \mathrm{AU}$ and reach up to $1000 \mathrm{AU}$.

The unshadowed part of the flaring disk reprocesses less radiation than the disk's inner rim, so that the spectrum has a strong 3 micron bump and falls off (in $v F_{v}$ ) as one goes to longer wavelengths. This conclusion is very similar to the conclusion drawn by Meeus et al. (2001) that the group IIa sources have no flaring disk. But there is an important difference. Our models have very small flaring disks, but they are certainly not vanishingly small. The 10 micron feature is still produced entirely by the compact flaring disk itself. Only the long wavelength part is suppressed by truncating the disk at small radii.

What is suspect about these fits is the fact, that we need a very specific location at which to truncate the disk. For the production of the silicate emission features, the disk needs to flare out of the shadow a little bit, but we cannot allow the disk to reach beyond $20 \mathrm{AU}$ because then the corresponding surface layer and midplane components would lead to a group I-like SED. What is truncating these disks at exactly the right radius? In particular, what is responsible for the striking differences between group Ia and group IIa? The near IR region as well as the submm fluxes of both groups are similar, the distribution on the sky and the absence of a nearby star forming region is the same for both. Why do the group IIa disk either collapse or shrink (without loosing mass) to very small sizes? We conclude that while the DDN model is capable of fitting the SEDs of group IIa sources very well, the model parameters derived seem difficult to understand.

\section{Summary}

In this paper we investigated the SEDs of the sample of 13 isolated Herbig Ae stars of Meeus et al. (2001) in terms of a passive irradiated flaring disk model. We find that for most of the stars the general shape of the SEDs can be naturally reproduced by the model. This adds evidence to the idea that the infrared excess of these stars is produced by a circumstellar disk, without need for additional circumstellar components. The conspicuous 3-micron bump in these SEDs, which until recently was considered an enigma, can be naturally explained as the emission from the inner rim of these disks. All of these disks have an inner rim emitting at $1500 \mathrm{~K}$ due to the fact that dust evaporation removes the dust (and therefore the opacity) at radii smaller than about half an AU. The disk picture is therefore a natural explanation for the infrared excess of most of these stars.

The three different types of spectra identified by Meeus et al. can, to a certain extent, be interpreted in terms of disk geometry. Their group Ia sources can be explained by large disks, some of which have cleared-out inner regions. The group IIa sources can be well fitted by compact disks with outer radii in the order of 10 to $50 \mathrm{AU}$. However, it is not clear 
what could cause such small disk radii in combination with the high surface densities needed to explain the disk mass. A collapsed outer disk which is not flaring may therefore be a more attractive scenario, which we will be exploring in a future publication. Only the group $\mathrm{Ib}$ sources, in which the 10 micron silicate feature is completely absent, seem to defy explanation in geometrical terms only. However, it is possible that additional effects like grain settling and disk collapse may help to resolve these differences.

Note added in proof: It has come to our attention that the $1.2 \mathrm{~mm}$ flux of HD 100453 plotted in Fig. 4, row 1 and 2 is incorrect. the correct flux is $0.23 \mathrm{Jy}$ ( $\mathrm{S}$. Wolf, private communication), and the corresponding value for $\log v F_{v}$ is -12.24 . This higher flux gives additional support to our fit 2 for this star, involving large grains.

Acknowledgements. We would like to thank A. Natta for stimulating discussions related to this paper, partly during a very pleasant working visit of $\mathrm{CD}$ to Arcetri. We would like to thank G. Meeus and M. van den Ancker for providing us with electronic version of their observed and dereddened data. CD and RW acknowledge the financial support from NWO Pionier grant 6000-78-333. CPD acknowledges support from the European Commission under TMR grant ERBFMRX-CT98-0195 ("Accretion onto black holes, compact objects and prototars").

\section{References}

Artymowicz, P., \& Lubow, S. H. 1996, ApJ, 467, L77

Aumann, H. H., Beichman, C. A., Gillett, F. C., et al. 1984, ApJ, 278, L23

Beckwith, S. V. W., Henning, T., \& Nakagawa, Y. 2000, Protostars and Planets IV, 533

Bogaert, E. 1994, Ph.D. Thesis

Bouwman, J., de Koter, A., Dominik, C., \& Waters, L. B. F. M. 2001, A\&A, 375, 950

Bouwman, J., de Koter, A., van den Ancker, M. E., \& Waters, L. B. F. M. 2000, A\&A, 360, 213

Bouwman, J., Meeus, G., de Koter, A., et al. 2002, A\&A

Calvet, N., D'Alessio, P., Hartmann, L., et al. 2002, ApJ, 568, 1008

Chiang, E. I., \& Goldreich, P. 1997, ApJ, 490, 368

Chiang, E. I., Joung, M. K., Creech-Eakman, M. J., et al. 2001, ApJ, 547,1077

di Francesco, J., Evans, N. J., Harvey, P. M., Mundy, L. G., \& Butner, H. M. 1994, ApJ, 432, 710

di Francesco, J., Evans, N. J., Harvey, P. M., Mundy, L. G., \& Butner, H. M. 1998, ApJ, 509, 324
Draine, B. T., \& Lee, H. M. 1984, ApJ, 285, 89

Dullemond, C. P., Dominik, C., \& Natta, A. 2001, ApJ, 560, 957

Dutrey, A., Guilloteau, S., Prato, L., et al. 1998, A\&A, 338, L63

Finkenzeller, U., \& Mundt, R. 1984, A\&AS, 55, 109

Grady, C. A., Devine, D., Woodgate, B., et al. 2000, ApJ, 544, 895

Grady, C. A., Polomski, E. F., Henning, T., et al. 2001a, AJ, 122, 3396

Grady, C. A., Woodgate, B., Gull, T., et al. 2001b, Amer. Astron. Soc. Meet., 198, 77.16

Hayashi, C. 1981, Progress of Theoretical Physics Supplement, 70, 35 Henning, T., Burkert, A., Launhardt, R., Leinert, C., \& Stecklum, B. 1998, A\&A, 336, 565

Herbig, G. H. 1960, ApJS, 4, 337

Laor, A., \& Draine, B. T. 1993, ApJ, 402, 441

Malfait, K., Bogaert, E., \& Waelkens, C. 1998, A\&A, 331, 211

Malfait, K., Waelkens, C., \& Vandenbussche, B. 1997, Ap\&SS, 255, 43

Mannings, V., \& Sargent, A. I. 1997, ApJ, 490, 792

Mannings, V., \& Sargent, A. I. 2000, ApJ, 529, 391

Marsh, K. A., Van Cleve, J. E., Mahoney, M. J., Hayward, T. L., \& Houck, J. R. 1995, ApJ, 451, 777

Meeus, G., Bouwman, J., Dominik, C., Waters, L. B. F. M., \& de Koter, A. 2002, A\&A

Meeus, G., Waters, L. B. F. M., Bouwman, J., et al. 2001, A\&A, 365, 476

Millan-Gabet, R., Schloerb, F. P., \& Traub, W. A. 2001, ApJ, 546, 358

Miroshnichenko, A., Ivezić, Ž., Vinković, D., \& Elitzur, M. 1999, ApJ, 520, L115

Natta, A., Prusti, T., Neri, R., et al. 2001, A\&A, 371, 186

O’Dell, C. R. 1998, AJ, 115, 263

Pantin, E., Waelkens, C., \& Lagage, P. O. 2000, A\&A, 361, L9

Prusti, T., Natta, A., \& Palla, F. 1994, A\&A, 292, 593

Qi, C., Blake, G. A., \& Sargent, A. I. 1999, in Science with the Atacama Large Millimeter Array (ALMA)

Scott, A., \& Duley, W. W. 1996, ApJS, 105, 401

Servoin, J. L., \& Piriou, B. 1973, Phys. Stat. Sol., 55, 677

Strom, S. E., Strom, K. M., Yost, J., Carrasco, L., \& Grasdalen, G. 1972, ApJ, 173, 353

Thamm, E., Steinacker, J., \& Henning, T. 1994, A\&A, 287, 493

The, P. S., de Winter, D., \& Perez, M. R. 1994, A\&AS, 104, 315

van den Ancker, M. E., de Winter, D., \& Tjin A Djie, H. R. E. 1998, A\&A, 330, 145

van den Ancker, M. E., Meeus, G., Cami, J., Waters, L. B. F. M., \& Waelkens, C. 2001, A\&A, 369, L17

van den Ancker, M. E., The, P. S., Tjin A Djie, H. R. E., et al. 1997, A\&A, 324, L33

Waelkens, C., Bogaert, E., \& Waters, L. B. F. M. 1994, in The Nature and Evolutionary Status of Herbig Ae/Be Stars, ASP Conf. Ser., 62, 405

Waters, L. B. F. M., \& Waelkens, C. 1998, ARA\&A, 36, 233 